\title{
Development of a Crosslink Channel Simulator
}

\author{
Chris Hunt, Carl Smith \\ General Dynamics Decision Systems \\ 8201 E. McDowell Rd. \\ Scottsdale, AZ 85257 \\ 480-675-1097 \\ chris.hunt@gdds.com
}

\author{
Rich Burns \\ NASA Goddard Space Flight Center \\ Greenbelt, MD 20771 \\ 301-286-9818 \\ rich.burns@nasa.gov
}

\begin{abstract}
Distributed Spacecraft missions are an integral part of current and future plans for NASA and other space agencies. Many of these multi-vehicle missions involve utilizing the array of spacecraft as a single, dispersed instrument requiring communication via crosslinks to achieve mission goals. NASA's Goddard Space Flight Center (GSFC) is developing the Formation Flying Test Bed (FFTB) to provide a hardware-in-the-loop simulation environment to support mission concept development and system trades with a primary focus on Guidance, Navigation, and Control (GN\&C) challenges associated with spacecraft formation flying. The goal of the FFTB is to reduce mission risk by assisting in mission planning and analysis, providing a technology development platform that allows algorithms to be developed for mission functions such as precision formation navigation and control and time synchronization. The FFTB will provide a medium in which the various crosslink transponders being used in multi-vehicle missions can be integrated for development and test; an integral part of the FFTB is the Crosslink Channel Simulator (CCS). The CCS is placed into the communications channel between the crosslinks under test, and is used to simulate on-mission effects to the communications channel such as vehicle maneuvers, relative vehicle motion, or antenna misalignment. The CCS is based on the Starlight ${ }^{\mathrm{TM}}$ software programmable platform developed at General Dynamics Decision Systems and provides the CCS with the ability to be modified on the fly to adapt to new crosslink formats or mission parameters.
\end{abstract}

This paper briefly describes the Formation Flying Test Bed and its potential uses. It then provides detail on the current and future development of the Crosslink Channel Simulator and its capabilities.

Keywords - Crosslink, Formation Flying, Space Electronics, Software Defined Radio, Transponder ${ }^{1}$

\section{TABLE OF CONTENTS}

1. INTRODUCTION 1

2. THE NASA FORMATION FLYING TEST BED ..................... 3

3. CROSSLINK CHANNEL SIMULATOR (CCS) .......................... 4

4. FutURe (PlanNed) CAPABILITIES................................... 6

${ }^{1}$ 0-7803-8155-6/04/\$17.00@ 2004 IEEE

IEEEAC paper \#1376, Version 5, Updated December 22, 2003

\author{
5. SUMMARY \\ REFERENCES \\ BIOGRAPHY \\ Acronym List \\ CCS: Crosslink Channel Simulator \\ DSP: Digital Signal Processing \\ DSS: Distributed Space Systems \\ ENV: Environment computer \\ FFTB: Formation Flying Testbed \\ GN\&C: Guidance, Navigation, and Control \\ GPS: Global Positioning System \\ GPSSG: GPS Signal Generator \\ GSFC: Goddard Space Flight Center \\ NASA: National Aeronautics and Space Administration \\ PN: Pseudorandom Noise \\ RF: Radio Frequency \\ UDP: Universal Datagram Protocol
} 7

\section{INTRODUCTION}

In the past, NASA's space-based missions have been organized around a single, typically large spacecraft carrying out all of the mission functions. As mission requirements grow to include science output that a single spacecraft alone cannot provide, this paradigm is changing to incorporate missions that utilize multiple spacecraft working as a single observing system. For example, some Distributed Space Systems (DSS) missions will rely on arrays of distributed spacecraft to form a single distributed aperture, using long baselines and interferometry to observe distant objects and events in great detail. A single spacecraft can only achieve the spatial diversity on the scale of its own structure, whereas the spacecraft array can form an effective aperture bounded only by the (engineering) limits on intersatellite separations. Other missions will utilize distributed in-situ measurements in order to distinguish spatial and temporal variations in the space environment. For example, the Magnetosphere MultiScale (MMS) mission [1] will utilize a constellation of four spacecraft to make measurements of the three-dimensional structure and dynamics of the key boundary regions of the Earth's mágnetosphere. 


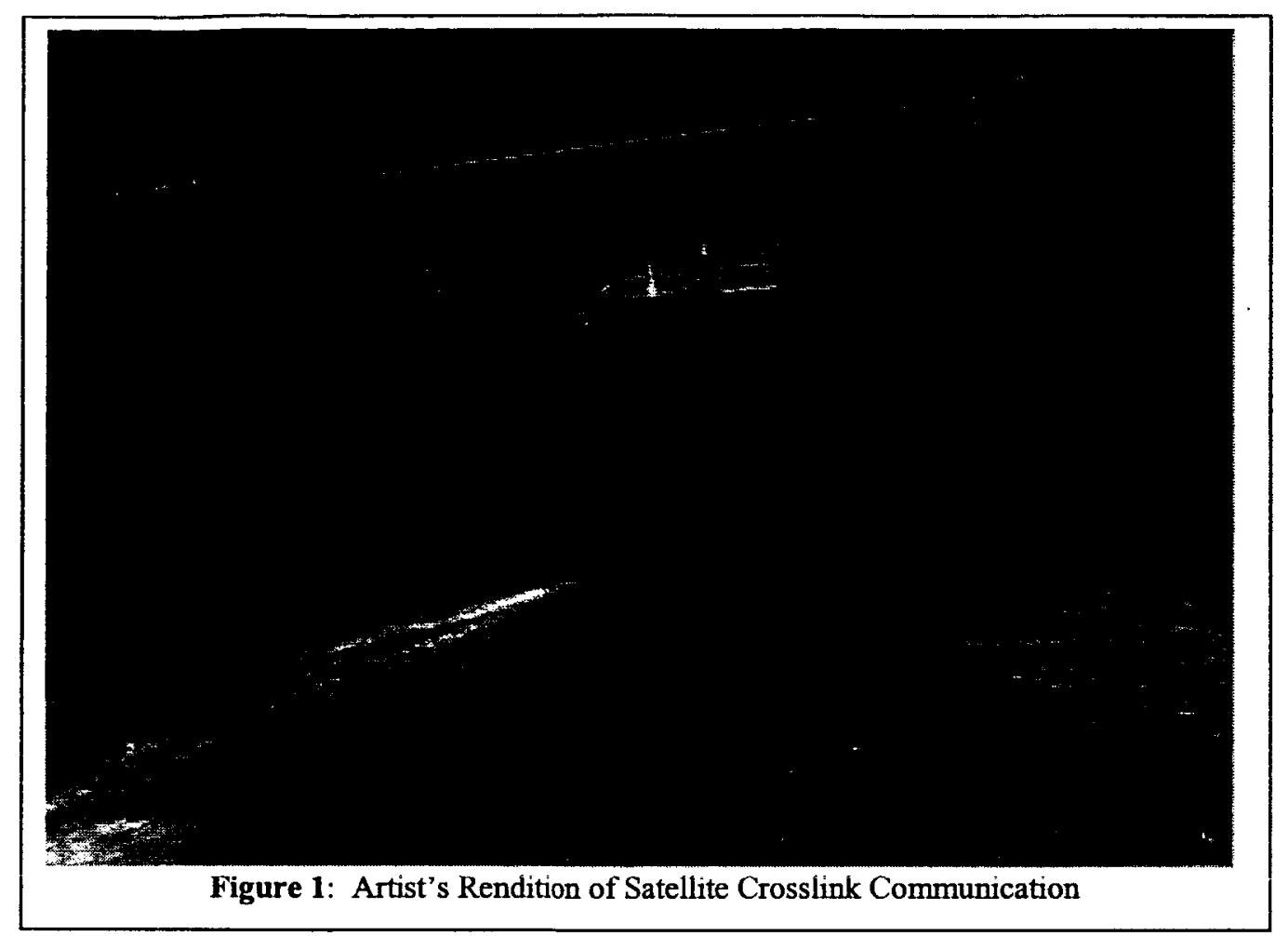

DSS missions also have the potential to reduce mission costs. Commercial spacecraft production will be able to reuse designs, components, and manufacturing that will make for smaller, less expensive and more reliable spacecraft. Also, if one unit fails the mission can still proceed with reduced capacity, a concept often referred to as graceful degradation.

Some of the future missions including constellations or formations of spacecraft planned by NASA are:

- Magnetosphere MultiScale (MMS) [1]

- Geospace Electrodynamic Connections (GEC) [2]

- Magnetospheric Constellation (MagCon) [3]

- Constellation-X [4]

- Terrestrial Planet Finder (TPF) [5]

- Laser Interferometer Space Antenna (LISA) [6]

- Stellar Imager (SI) [7]

- MAXIM \& MAXIM Pathfinder [8]

To achieve the science goals for these missions, each spacecraft in the constellation may carry a set of instrumentation which collects data that must be sent back to the mission operators on Earth. In some cases, only one of the spacecraft has a high-gain antenna that is used to transmit the mission data back to Earth, and the data from the other space vehicles in the constellation must be passed over so it can be transmitted as well.

Furthermore, some of these missions require the spacecraft to fly in formation, that is, to control their relative position and/or attitude. Formation flying generally requires the estimation of relative position, velocity, and attitude, which is also called relative navigation. In such a system requiring relative navigation and control, the intersatellite communications device (crosslink) can serve a dual role: 1) to provide a means of intersatellite communications, which is required to a share measurements and control messages such as maneuver commands and 2) to make direct measurement of the scalar distance between spacecraft, also called ranging. Through these roles, the crosslink becomes a critical technology for successfully closing the control loop for formation flying systems. Figure 1 shows an artist's rendition of a satellite formation utilizing crosslink capabilities.

Consequently, it is important that the FFTB acquire the capability to insert crosslink hardware into its simulation. Of course, crosslinks can be connected directly to one another in the FFTB using coaxial cables and attenuators, and this configuration is useful to establish a communication path through the crosslink hardware and to test the interface between the crosslinks and the flight software. However, direct connection of the crosslinks does not account for effects on the signal, such as delay, Doppler frequency shift, and attenuation, which are functions of the satellites' states (position, velocity, attitude) and geometry. The Crosslink Channel Simulator (CCS) is inserted into the communication paths between the crosslinks to introduce these effects. Introduction of crosslink hardware and statebased signal effects to the FFTB will provide a realistic representation of intersatellite communications and ranging, enabling integration and test of formation flying navigation and control algorithms in a high fidelity, hardware-in-theloop simulation environment. 
This paper discusses the development of the FFTB, its uses and capabilities, as well as the details of the CCS including future upgrades for the system. enabling technology. The FFTB seeks to provide this simulation capability; its role includes investigation of DSS mission feasibility, definition of engineering requirements,

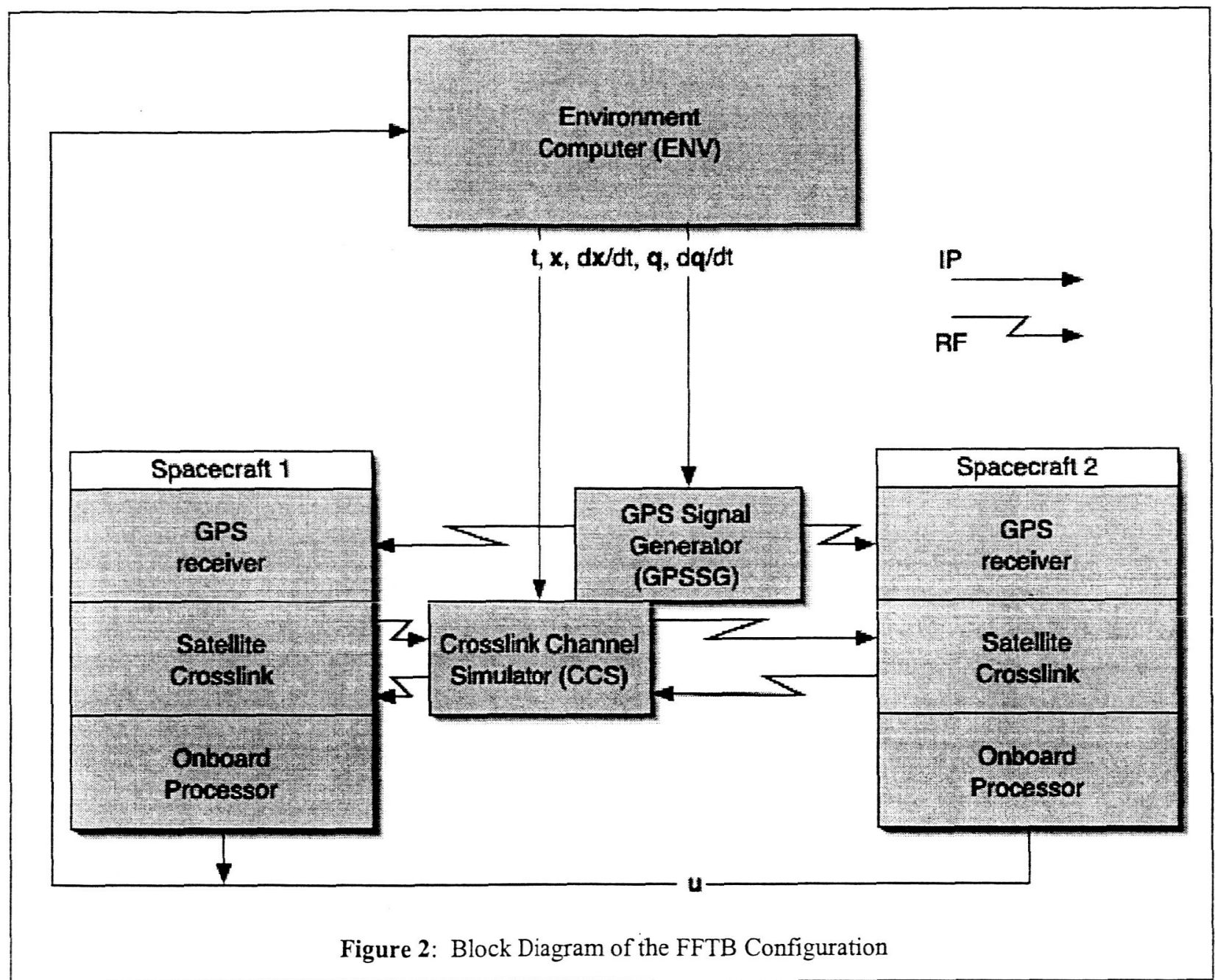

\section{The Formation Flying Testbed}

Many of these DSS missions require control of the satellites' relative position and/or attitude; these missions will be referred to as formation flying missions. Among the formation flying missions, there exists another subset whose relative control requirements are so demanding that they will require nearly continuous control; these are termed precision formation flying missions. NASA has an abundance of DSS missions on its roadmap, and several examples have already been mentioned in the previous section. Among these, MMS is an example of a formation flying mission, and Stellar Imager and Maxim are both precision formation flyers.

The fact that formation flying systems are significantly different and potentially more complicated than the traditional single satellite systems provides motivation to build hardware-in-the-loop simulation capabilities such that these systems can be conceived and developed within a realistic framework and with an eye toward identifying maturation of required sensor and actuator hardware and GN\&C software, and validation of GN\&C system performance.

A core tenet in the design of the FFTB has been the development of the capability to include GN\&C hardware in the simulation loop. GPS receivers were the first to be included in FFTB simulations due to the availability of GPS signal generators as well as receiver hardware. While simulations of GPS-based DSS missions have been useful, it has become clear that the hardware-in-the-loop simulation of formation flying systems must grow to encompass the satellite crosslinks. Crosslinks provide two major functions from an engineering perspective, namely intersatellite communication and intersatellite range measurement. Intersatellite communications enable the distribution of satellite commands and the sharing of engineering data. Intersatellite ranging is a key measurement for input to the relative navigation system.

The CCS, detailed in the next section, is the mechanism by which the FFTB is incorporating crosslink. It is envisioned 
that the CCS will enhance the FFTB's current closed loop formation control simulation by enabling the loop to be closed through real crosslink hardware.

A schematic diagram of the FFTB is shown in Fig 2. The Environment computer is responsible for the numerical integration of the satellites' equations of motion thereby generating truth trajectories including time, position, velocity, attitude, and attitude rates $(t, x, d x / d t, q, d q / d t)$. This trajectory information is then delivered in real time to the GPS Signal Generator (GPSSG) and the CCS. Armed with the knowledge of the receivers' states, the GPSSG generates a Radio Frequency (RF) signal that simulates the received signal from the GPS constellation complete with time delay and Doppler shifting. Similarly, the CCS provides RF signal to the crosslinks which represents the signal which would be received by the crosslinks during their space mission including time delay, Doppler frequency shifts, and attenuation based on the current relative states of the transmitting and receiving satellites. The on-board processor hosts the flight software including the navigation and control systems. GPS measurements are delivered to the on board processor for inclusion in the navigation processing. Depending on the navigation and control schemes, the processor may then send out data or commands to the other satellites through the crosslinks. Maneuver commands (u) are also fed back to the Environment computer for integration into the equations of motion. Consequently, the crosslink hardware is in the critical path for closing the formation control loop and underscores the motivation for including it in the FFTB simulation.

\section{Crosslink Channel Simulator (CCS)}

The development of the CCS is progressing in two phases. In the first phase, General Dynamics Decision Systems has designed and built a prototype CCS for the FFTB. The prototype CCS, built in fiscal year 2003, supports an RF crosslink frequency of $2448 \mathrm{MHz}$ and provides partial RF functionality. That is, it operates in a "talk-only" mode in which it is capable of generating the RF signal for the receiver without "listening" to the signal from the transmitter. This is accomplished by adding data content to the messages the CCS receives over Internet Protocol (IP) socket connection from the ENV. While the prototype CCS enables the introduction of hardware-in-the-loop communications and ranging to the FFTB, its "talk-only" limitations mean that the transmit functionality of the crosslink hardware is not utilized in the simulation.

The second stage of CCS development, occurring in fiscal year 2004, will enable 1) full RF functionality i.e., the ability to receive RF signal from the source crosslink and transmit modified RF signal to the destination crosslink, 2) the capability to support a network of four crosslinks, and 3) communication over a wider range of frequency bands, ideally making it compatible with all existing crosslink transponders.
The space-to-space signals transmitted between crosslinks will experience several communications channel effects which are duplicated in simulation by the CCS. These are: Doppler shift caused by the relative motion of the spacecraft; signal attenuation caused by free space loss between the two spacecraft or other losses such as antenna mispointing; and signal delay caused simply by the time of signal transit between the two spacecraft. The CCS simulates all three of these channel effects very precisely, with signal delay simulated down to $1 \mathrm{~cm}$ of range accuracy, or about 33 picoseconds for a typical S-band crosslink. Doppler shift is simulated down to less than $1 \mathrm{~mm} / \mathrm{s}$, and signal attenuation is controlled to $0.1 \mathrm{~dB}$. The $1 \mathrm{~cm}$ accuracy requirement over the entire possible range of spacecraft separation distances (from 0 to $6400 \mathrm{~km}$ or more) drove the design to perform baseband signal processing to implement the channel effects instead of direct RF/IF sampling. Delaying the crosslink signal by $33 \mathrm{pS}$ (corresponding to $1 \mathrm{~cm}$ of range difference) corresponds to approximately a 30 $\mathrm{GHz}$ sampling rate which is not available with current technology. Therefore, advanced signal processing techniques were used to simulate the crosslink signal ranges down to the $1 \mathrm{~cm}$ resolution.

In the second phase of development, the CCS must have two-way communications with both spacecraft transponders; it must be able to receive and transmit the crosslink signal structure, in effect being able to act as a two-way crosslink transponder itself. After one crosslink transmits its RF signal to the CCS, the CCS must demodulate the signal, stripping it of its carrier and PN code, induce the appropriate channel effects, remodulate the signal onto the appropriately modified RF carrier, and transmit the modified signal to the receiving crosslink transponder.

One of the major requirements of the CCS was that it be able to communicate with a wide variety of crosslink transponder devices using their own transmit and receiver frequencies, message formats, and data rates. This requirement drove the $\mathrm{CCS}$ design to include a reconfigurable hardware system that can be changed via software to transmit and receive a new signal format. This requirement was met by using the General Dynamics software programmable communications processing platform known as the StarLight ${ }^{\mathrm{TM}}[9]$. The StarLight architecture implements an optimized combination of RF signal processing, ASIC-based digital signal processing, and software signal processing and control. The StarLight supports varied application types (from GPS to high-speed crosslinks), signaling formats, and is reconfigurable "on the fly."

\section{System Overview}

Figure 3 shows the system block diagram of the protoype CCS. The major components that comprise the CCS hardware are two RF boards, a digital board, several 


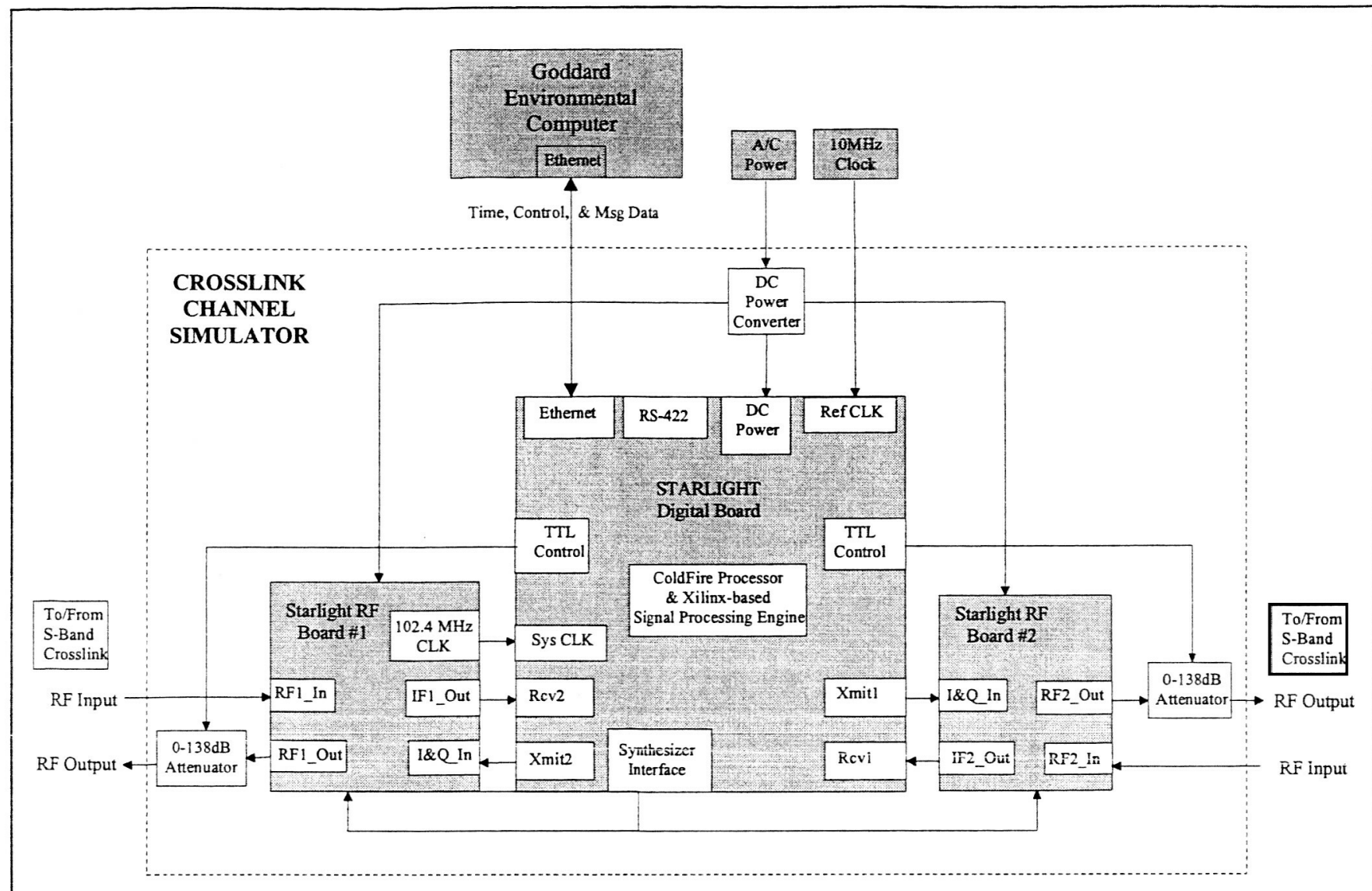

Figure 3: Crosslink Channel Simulator System Block Diagram

digitally-programmable attenuators and a power supply. The digital and RF cards are based on General Dynamics software programmable communications processing platform known as the StarLight. These cards and the other supporting hardware are installed into a 19" Rackmount chassis for installation into the FFTB at GSFC. Figure 4 shows a picture of the prototype CCS box in the rackmount chassis. The CCS accepts an external reference $10 \mathrm{MHz}$ clock and a 1 Pulse Per Second (1 PPS) input to synchronize it with the rest of the FFTB equipment.

Each of the two CCS RF cards provides one RF receiver input and one RF transmitter output via $\mathrm{N}$-type connectors on the front cover of the CCS rackmount chassis. The Sband RF downconverter chain contained on each board provides a downconverted second IF frequency output to the CCS digital card. To facilitate the transmit function, the RF cards accept baseband in-phase (I) and quadrature (Q) signals from the CCS digital card modulate these signals onto RF carriers. The frequency synthesis for the receiver's local oscillator signals, the transmitter carrier frequency and the clock signal for the StarLight card are all provided by the RF assembly and are phase locked to the externally supplied $10 \mathrm{MHz}$ reference oscillator input. A $102.4 \mathrm{MHz}$ clock is generated on both RF cards, and this clock signal is routed from one of the RF boards to the digital card to be used as the system clock.
The CCS digital card provides all of the command and data handling, any necessary parameter calculations, transmitter and receiver control, and other signal processing functions. Commands to the CCS through its Ethernet port are interpreted and processed by the onboard ColdFire ${ }^{\circledR}$ processor. These commands can contain CCS configuration commands or requests and simulation parameters such as time delay, attenuation, and Doppler shift.

The digital card provides analog to digital converter (ADC) inputs connected to the IF outputs from each of the two receiver channels on the CCS RF cards. Each ADC samples one of the IF signals at the system clock rate and inputs the samples to the Digital Signal Processing (DSP) gateware where appropriate techniques are applied to extract and process the signal modulated onto the received carrier.

The transmitter portion of the CCS digital card accepts a single data stream provided by the processor after it has been extracted from the received messages. Depending upon the current configuration of the CCS, this data stream could be re-formatted (e.g. from NRZ-L to NRZ-M), encoded, interleaved, or combined with a Pseudorandom Noise (PN) code. The CCS then buffers the data, modulates it onto a baseband carrier at the required Doppler offset, and then sends it to the RF transmitters at a specific time so the data appears to be delayed and channel-modified to the receiving crosslink. 


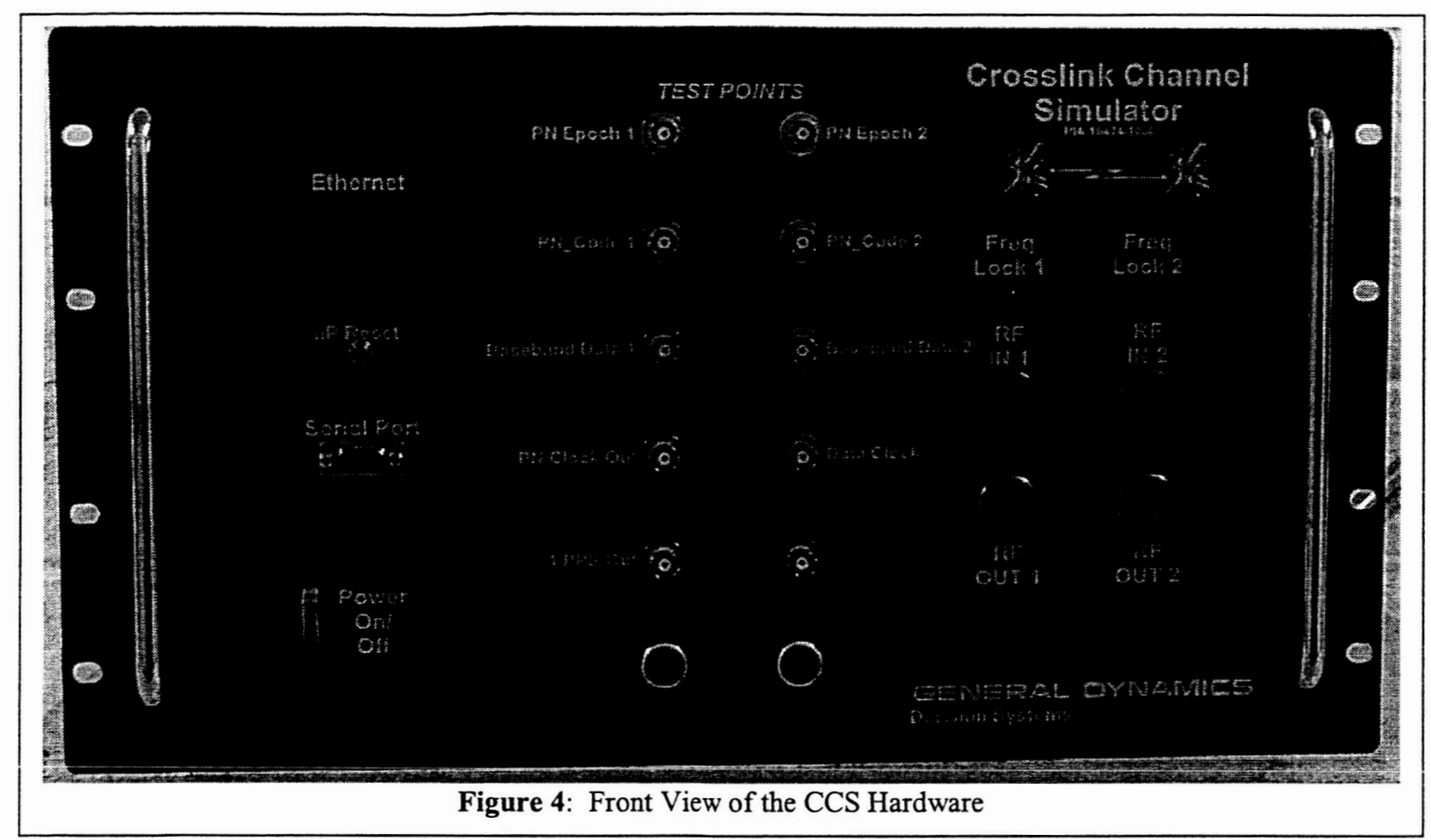

\section{Software Architecture}

The software developed for the CCS provides an interface to configure, monitor, and manage channel data during simulations run on the FFTB. Using VxWorks ${ }^{\circledR} 5.5$ kernel from Wind River Systems, Inc. for the Coldfire CPU, the CCS software is designed to react to the needs of the configured hardware to provide it both the message buffer data stream as well as computed signal manipulations provided by the ENV.

The ENV provides the channel parameters with each message to be transmitted. These parameters include acceleration, Doppler, signal delay, and attenuation. The channel-processing task takes these parameters and generates data packages that will be used by the associated interrupt service routine to provide control signals to the transmitter hardware. The acceleration and Doppler parameters are used in conjunction to provide updates to the transmitter frequency at a specified interval. The ENV is responsible for maintaining the continuity of all parameters between each message packet.

The pilot development of the CCS software occurred over a 6-week period. Software was developed using $C$ on VxWorks Tornado for Windows 2000 workstations. The VxWorks Simulator, provided with this platform, provided an environment to develop software as the hardware was completed. This allowed the engineers to develop and test all tasking, inter-process communication, and message processing behaviors prior to integration on the StarLight Platform. CCS was able to leverage the UDP utilities developed during a previous StarLight-based project [9].
Hardware/Software integration cycle was short due to: 1) Stable hardware definitions and memory map; 2) VxWorks Tornado fast cycle time for Test, Debug, Correct; and 3) Significant testing with the VxWorks Simulator. An HP VEE application was also developed to simulate the ENV and validate the UDP communication formats.

\section{Future (Planned) Capabilities}

Figure 5 shows the basic block diagram for configuration of the CCS after phase two of the development. As mentioned previously, this configuration upgrades the current CCS to include the ability to place up to four crosslink transponders simultaneously into the FFTB. The future CCS will include the following:

- Full RF functionality

- Four transmitters

- Four receivers

- Routing between the four communication paths

- Greater frequency agility

With this set of upgrades, the FFTB will move beyond the simple point-to-point communications and ranging interaction of a two-satellite formation. Support for four crosslinks will enable more complex and realistic communications and ranging schemes. Full RF functionality of the CCS will allow for the exercise of the crosslink's transmit function, building more confidence in the realism of the simulation. 


\section{SUMMARY}

The GSFC Formation Flying Testbed will provide a facility to simulate the crosslink communications and relative navigation between spacecraft on the ground, prior to launch. Since many future NASA programs include formations of spacecraft relying on intersatellite communications, the FFTB will become an increasingly important tool in the success of these missions.

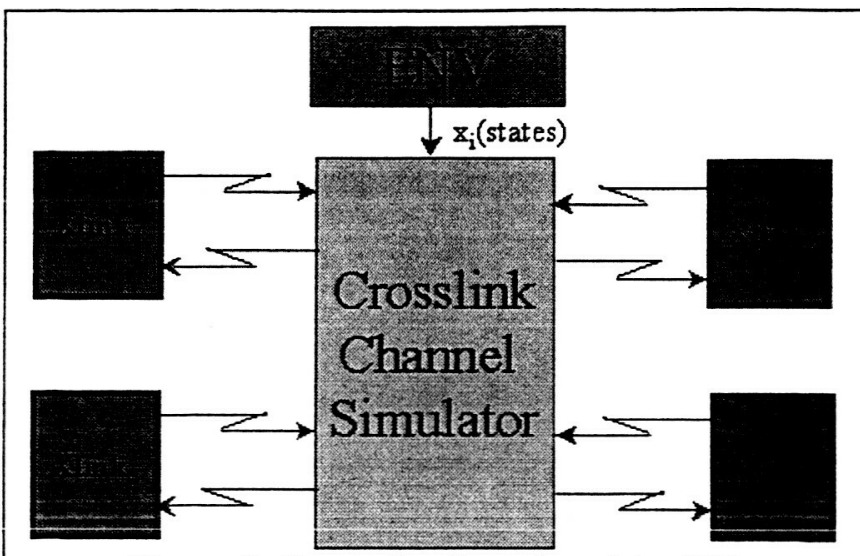

Figure 5: Future Configuration of the CCS

The Crosslink Channel Simulator developed by General Dynamics is one of the major enablers of the FFTB. It provides the FFTB with the ability to simulate the physical effects of the mission environments, including Doppler shift, signal attenuation, and delay. Because the CCS was developed using the Starlight ${ }^{\mathrm{TM}}$ reconfigurable hardware platform, it can easily be commanded via software to interface with a wide variety of crosslink transponder hardware. This allows NASA to evaluate many crosslink options for each mission with one set of FFTB equipment.

The initial development of the CCS has been demonstrated in the FFTB facility at GSFC. In 2004, development of the CCS will continue towards its future configuration to enable up to four crosslink transponders to simultaneously operate in the FFTB.

Starlight ${ }^{\mathrm{TM}}$ is a trademark of General Dynamics, ColdFire ${ }^{\circledR}$ is a registered trademark of Motorola, Inc., and VxWorks $\AA$ is a trademark of Wind River Systems, Inc.

\section{REFERENCES}

[1] Magnetosphere MultiScale Mission Web Site, http://stp.gsfc.nasa.gov/missions/mms/mms.htm

[2] Geospace Electrodynamic Connections (GEC) Web Site, http://stp.gsfc.nasa.gov/missions/gec/gec.htm

[3] Magnetospheric Constellation Web Site, http://stp.gsfc.nasa.gov/missions $/ \mathrm{mc} / \mathrm{mc}$.htm

[4] Constellation-X Web Site, http://constellation.gsfc.nasa.gov/docs/main.html

[5] Terrestrial Planet Finder Web Site, http://tpf.jpl.nasa.gov
[6] Laser Interferometry Space Antenna (LISA) mission Web Site, http://lisa.jpl.nasa.gov/

[7] Stellar Imager (SI) mission Web Site, http://hires.gsfc.nasa.gov/ si/

[8] Micro-Arcsecond X-ray Imaging Mission (MAXIM) Web Site, http://maxim.gsfc.nasa.gov/

[9] C. Long, C. Hunt, M. Siemon, "Applications for a Programmable Space Platform," 2003 IEEE Aerospace Conference Proceedings, March 8-15, 2003.

\section{BIOGRAPHY}

Chris Hunt is a communications systems engineer at GDDS working with spaceborne communications systems including transponders and GPS receivers. Recently Chris has specialized in the modeling and simulation of the signal processing used in the systems developed at GDDS for

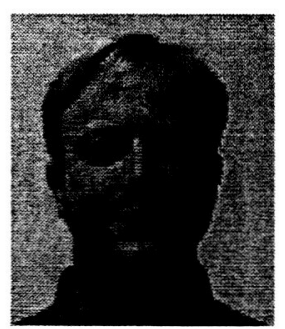
spaceborne applications. He has developed RF and signal processing subsystems for several spacecraft missions and is currently working on an upgrade for the International Space Station. He holds both BSEE and MSEE degrees from Kansas State University.

Carl Smith Carl Smith is a principal software engineer at GDDS working with spaceborne communications including the Iridium satellite constellation and GPS receivers. Recently, Carl has worked on upgrades to the ISS Space Station communication system and several high availability telephony projects. He holds a BSEE from Polytechnic University and a MSEE from Drexel University.

Rich Burns has served as the lead engineer in the Formation Flying Testbed at NASA's Goddard Space Flight Center since 2001. Prior to working at NASA, Rich worked as an engineer and group lead at the Air Force Research Laboratory's Space Vehicles Directorate. He holds a M.S.

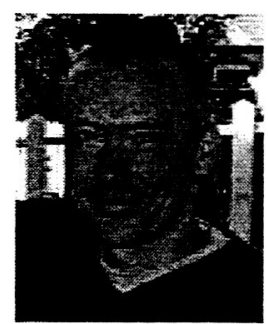
from Stanford and a B.S. from Notre Dame in Mechanical Engineering. 